\title{
CONTROLABILIDAD APROXIMADA PARA UN SISTEMA DE ECUACIONES DIFERENCIALES PARCIALES EN UN DOMINIO NO CILINDRICO
}

Sea $\Omega$ un abierto acotado de $\mathbb{R}^{n}$, el cual sin pérdida de generalidad podemos suponer conteniendo el origen de $I R^{n}$, con frontera $\Gamma$ bien regular y $Q_{t}=\Omega \times[0, T[(T>0)$ un cilindro de $I R^{n+1}$, con frontera lateral $\left.\sum_{T}=\Gamma \times\right] \mathrm{b}, T[$. Consideremos la función

$$
t \mapsto K(t), \text { de }[0, T] \rightarrow I R^{n^{2}} .
$$

es decir $K(t)$ es la matriz de orden $n \times n$

Supongamos $K(t)$ ortogonal y continuamente diferenciable para cäda $t$.

Definimos el conjunto

$$
\Omega_{t}=\{x=K(t) y ; y \in \Omega\} \quad(0 \leq t \leq T)
$$

cuya frontera es denotada por $\Gamma_{t}$. Representemos por $\hat{Q}_{T}$ el dominio no cilíndrico

con frontera lateral

$$
\hat{Q}_{T}=\bigcup_{0<t<T} \Omega_{t} \times\{t\}
$$

$$
\hat{\Sigma}_{T}=\bigcup_{0<t<T} \Gamma_{t} \times\{t\}
$$

Sea $\omega$ un subconjunto abierto no vacío de $\Omega_{0}$, tal que el cilindro $\left.q_{T}=\omega \times\right] 0, T[$ está contenido en $\hat{Q}_{T} \cdot \chi_{q_{T}}$ denota la función característica de $q_{T}$ y $\hat{\omega}(x, t)$ es la función control con soporte contenido en $q_{T}$.

En este trabajo estudiamos la controlabilidad aproximada para el siguiente sistema:

$$
\begin{aligned}
& u^{\prime}(x, t)-\Delta u(x, t)+\hat{a}(x, t) u(x, t)+\hat{\hat{b}}(x, t) \cdot \nabla u(x, t)=\hat{\omega}(x, t) \chi_{q_{T}} \text { en } \hat{Q}_{T} \\
& u(x, t)=0 \quad \text { sobre } \quad \hat{\Sigma}_{T} \\
& u(x, 0)=u_{0}(x) \quad \text { en } \quad \Omega_{0}
\end{aligned}
$$

donde $u(x, t)$ es la función vectorial $\left(u_{1}(x, t), \ldots, u_{n}(x, t)\right)$ con $(\mathrm{x}, \mathrm{t}) \in \hat{Q}_{T}$.

\footnotetext{
1 Centro Universitario de Palmas, Fundación Universidad de Tocantins, Caixa Postal 114. CEP 77000-000,
} Palmas-TO Brasilia - Brasil. 
Con el símbolo $\Delta$ representamos la matriz $\left(\Delta_{i j}\right)_{n \times n}$ de operadores definida por

$$
\Delta_{i j}=\mid \begin{array}{ccc}
\Delta & \text { para } & i \leq j \\
0 & \text { para } & i>j
\end{array}
$$

donde $\Delta$ es el operador Laplaciano; $\hat{a}(x, t)=\left(\hat{a}_{i j}(x, t)\right)_{n \times n}$ y $\overrightarrow{\hat{b}}(x, t)=\left(\overrightarrow{\hat{b}}_{i j}(x, t)\right)_{n \times n} \quad$ matrices tales que $\hat{a}_{i j}(x, t)=0$ para $i>j \quad$ y $\vec{b}_{i j}(x, t)=(0,0, \cdots, 0)$ para $i>j, \hat{a}_{i j}(x, t) \in L^{\infty}\left(Q_{T}\right)$, $\overrightarrow{\hat{b}}_{i j}(\cdot, t) \in\left[L^{\infty}\left(Q_{T}\right)\right]^{n}, \nabla u(x, t)=\left(\nabla u_{1}(x, t), \cdots, \nabla u_{n}(x, t)\right), \nabla$ es el operador gradiente

$$
\hat{\omega}(x, t)=\left(\hat{\omega}_{1}(x, t), \cdots, \hat{\omega}_{n}(x, t)\right)
$$

El presente trabajo está basado en resultados obtenidos por L. A. Medeiros [6] en los cuales se estudia la controlabilidad aproximada para el sistema (1) en el dominio no cilíndrico $\hat{Q}_{T}$ en el caso en que el sistema se reduce a una sola ecuación siguiendo las ideas de Fabre-PuelZuazua [2], Fabre [3], Lions [4]; sistemas, semejantes a (1) son considerados en Lions [5].

Transformaremos el sistema (1) en un sistema equivalente en dominio no cilíndrico mediante la aplicación

$$
\tau: \hat{Q}_{T} \rightarrow Q_{T}
$$

definido por $\tau(x, t)=\left(K(t)^{-1} x, t\right)$ con inversa

$$
\tau^{-1}: Q_{T} \rightarrow \hat{Q}_{T}
$$

dado por $\tau^{-1}(y, t)=(K(t) x, t)$. Así, haciendo $u(x, t)=v\left(K^{-1}(t) x, t\right), \quad v: Q \rightarrow I R$, $K^{-1}(t) x=y$ tenemos

$$
\begin{aligned}
& u^{\prime}(x, t)=v^{\prime}\left(K^{-1}(t) x, t\right)+\nabla v\left(K^{-1}(t) x, t\right) \cdot K^{-1}(t) K^{\prime}(t) y \\
& \nabla u(x, t)=\nabla v\left(K^{-1}(t) x, t\right) K^{-1}(t) \\
& \Delta u(x, t)=\Delta v\left(K^{-1}(t) x, t\right) \\
& \hat{a}_{i j}(x, t)=a_{i j}\left(K^{-1}(t) x, t\right) \\
& \overrightarrow{\hat{b}}_{i j}(x, t)=\overrightarrow{\hat{b}}_{i j}\left(K^{-1}(t) x, t\right) \\
& \hat{\omega}_{i}(x, t)=\omega_{i}\left(K^{-1}(t) x, t\right)
\end{aligned}
$$


Luego el sistema (1) queda transformado en

$$
\begin{aligned}
& v^{\prime}-\Delta v-\Delta v K^{-1}(t) K^{\prime}(t) y+a(y, t) v+\vec{b}(y, t) \nabla v K^{-1}(t)=\omega \chi_{q_{T}} \text { en } \quad Q_{T} \\
& v(y, t)=0 \quad \text { sobre } \quad \sum_{T} \\
& v(y, 0)=v_{0}(y) \quad \text { en } \quad \Omega
\end{aligned}
$$

donde

$$
\begin{aligned}
& a(y, t)=\left(a_{i j}(y, t)\right)_{n \times n}, \vec{b}(y, t)=\left(b_{i j}(y, t)\right)_{n \times n} \\
& \omega(y, t)=\left(\omega_{1}(y, t), \cdots, \omega_{n}(y, t)\right), \hat{q}_{T}=\left\{\left(K^{-1}(t) x, t_{0}\right),(x, t) \in q_{T}, 0<t<T\right\} . \\
& v=\left(v_{1}, \cdots, v_{n}\right), \quad v_{i}(y, t)=u_{i}(x, t) ; v_{0}(y)=u_{0}(y)
\end{aligned}
$$

Dado que $K(t)$ es ortogonal tenemos

$-\nabla v K^{-1}(t) K^{\prime}(t) y=K^{*^{\prime}}(t) K(t) y=y^{*} K^{-1}(t) K^{\prime}(t) \nabla v \quad$ y $\quad \nabla v K^{-1}(t)=K(t) \nabla v$ donde $A^{*}$ denota la matriz transpuesta de $A$.

Denotando nuevamente por $\vec{b}_{i j}$ a la suma $y^{*} K^{-1}(t) K^{\prime}(t) \delta_{i j}+\vec{b}_{i j} K(t)$ donde

$$
\begin{gathered}
\delta_{i j}=\mid \begin{array}{lrr}
1 & \text { si } & i=j \\
0 & \text { si } & i \neq j
\end{array}, \text {, tenemos que el sistema (2) queda aún transformado en: } \\
v^{\prime}-\Delta v+a(y, t) v+\vec{b}(y, t) \nabla v=\omega \chi_{q_{T}} \text { en } Q_{T} \\
v(y, t)=0 \quad \text { sobre } \quad \sum_{T} \\
v(y, 0)=v_{0}(y) \quad \text { en } \quad \Omega
\end{gathered}
$$

Soluciones en $Q_{T}$ y $\hat{Q}_{T}$

Sean $V$ y $H$ espacios de Hilbert con producto interno y norma representados por $((\cdot, \cdot)),\|\cdot\|$ y $(\cdot, \cdot),|\cdot|$. Supongamos $V \subset H$ con inmersión continua. Consideremos el espacio vectorial

$$
W(0, T ; V, H)=\left\{z \in L^{2}(0, T ; V), z^{\prime} \in L^{2}(0, T ; H)\right\}
$$

donde $z^{\prime}$ es la derivada en el sentido de las distribuciones. $W$ es un espacio de Hilbert con la norma:

$$
\|z\|_{W(0, T ; H)}^{2}=\|z\|_{L^{2}(0, T ; V)}^{2}+\left\|z^{\prime}\right\|_{L^{2}(0, T ; H)}^{2} .
$$


Este espacio de Hilbert tiene inmersión continua en el espacio de Banach $C^{0}([0, T] ; H)$; de este modo tiene sentido $z(0)$ y $z(T)$ para elementos de $W(0, T ; V, H)$.

Definición 1: La función $v: Q_{T} \rightarrow I R$ será llamada la solución fuerte del problema (3) si $\nu \in W\left(0, T ; H_{0}^{1}(\Omega)^{n} \cap H^{2}(\Omega)^{n}, L^{2}(\Omega)^{n}\right) y$

$$
v^{\prime}-\Delta v+a v+\vec{b} \nabla v=\omega \chi_{\hat{q}_{T}} \quad \text { c.s. } \quad \text { en } \quad Q_{T}
$$

y la condición inicial $v(y, 0)=v_{0}$.

Definición 2: La función $u: \hat{Q}_{T} \rightarrow I R$ será llamada solución fuerte del problema (1) si $u \in W\left(0, T ; H_{0}^{1}\left(\Omega_{t}\right)^{n} \cap H^{2}\left(\Omega_{t}\right)^{n}, L^{2}\left(\Omega_{t}\right)^{n}\right) y$

$$
u^{\prime}-\Delta u+\hat{a} u+\hat{\hat{b}} \nabla u=\hat{\omega} \chi_{q_{T}} \text { c.s. en } \quad \hat{Q}_{T}
$$

Espacios funcionales sobre $\hat{Q}_{T}$ son dados como en Milla-Limaco [7] .

Teorema 1: Dado $\omega \in L^{2}\left(0, T ; L^{2}(\Omega)^{n}\right)$ y $v_{0} \in H_{0}^{1}(\Omega)^{n}$ existe una única función $v: Q_{T} \rightarrow I R$ solución fuerte del problema (3). Esta solución tiene la siguiente regularidad:

$$
v \in C^{0}\left([0, T] ; H_{0}^{1}(\Omega)^{n}\right) .
$$

Demostración: Para una demostración usamos el método de Galerkin conforme a [1]. Observando que el sistema es triangular superior, las limitaciones son obtenidas a partir de la última línea en el problema aproximado.

Así, dada la limitación en la última línea usamos este resultado para limitar la penúltima línea y así sucesivamente. Para las limitaciones en la última línea ver [6].

Teorema 2: Dado $\hat{\omega} \in L^{2}\left(0, T ; L^{2}\left(\Omega_{t}\right)^{n}\right)$ y $u_{0} \in H_{0}^{1}\left(\Omega_{0}\right)^{n}$ existe una única función $u: \hat{Q}_{T} \rightarrow$ IR solución fuerte del problema (1). Esta solución tiene la siguiente regularidad:

$$
u \in C^{0}\left([0, T] ; H_{0}^{1}\left(\Omega_{t}\right)^{n}\right) .
$$

Demostración: Dado que $x=K(t) y$ tenemos $u(x, t)=v\left(K^{-1}(t) x, t\right) ; u_{0}(x)=v_{0}(y)$.

Así por el teorema 1 existe $v: Q_{T} \rightarrow \mathbb{R}$ solución fuerte de (3) tal que $v \in C^{0}\left([0, T] ; H_{0}^{1}(\Omega)^{n}\right)$.

Este resultado sigue de la equivalencia de los sistemas.

Así tenemos el siguiente resultado de controlabilidad aproximada para el sistema (1). 
Teorema 3: Dado $u_{0} \in H_{0}^{1}\left(\Omega_{0}\right)^{n}$ el conjunto de estados admisibles en el tiempo $T$

$$
\hat{R}_{L} x(T)=\left\{u(x, T) ; \hat{\omega} \in L^{2}\left(q_{T}\right)^{n}, \quad u \text { solución fuerte de }(1)\right\}
$$

es denso en $H_{0}^{1}\left(\Omega_{T}\right)^{n}$.

Dada la equivalencia de los problemas (1) y (3) será suficiente probar el siguiente lema

Lema 3.1: Dado $v_{0} \in H_{0}^{1}(\Omega)^{n}$ el conjunto de estados admisibles en el tiempo $T$

$$
R_{L}(T)=\left\{v(y, T) ; \omega \in \dot{L}^{2}\left(\hat{q}_{T}\right)^{n}, \quad v \text { solución fuerte de (3) }\right\}
$$

es denso en $H_{0}^{1}(\Omega)^{n}$.

Demostración: Sea $v_{d} \in H_{0}^{1}(\Omega)^{n}$, probaremos la existencia de una sucesión $\left(v_{k}(T)\right)_{k \in I N}$ tal que $v_{k}(T) \in R_{L}(T)$ y $v_{k}(T) \rightarrow v_{d}$ fuerte en $H_{0}^{1}(\Omega)^{n}$.

Por la linealidad de (3) bastará mostrar el lema para el caso en que $v_{0}=0$.

Para cada $k \in I N$ definimos el funcional

$$
J_{k}(\omega)=\frac{1}{2} \sum_{i=1}^{n} \int_{\hat{q}_{T}} \omega_{i}^{2}(y, t) d y d t+\frac{k}{2} \sum_{i=1}^{n}\left\|v_{i \omega}(T)-v_{i d}\right\|^{2}
$$

donde $v_{\omega}$ denota la solución fuerte de (3) para $\omega \in L^{2}\left(\hat{q}_{T}\right)^{n}$ y $v_{\omega}=\left(v_{1 \omega}, \cdots, v_{n \omega}\right)$, $v_{d}=\left(v_{1 d}, \cdots, v_{n d}\right)$.

Consideremos ahora el siguiente problema de minimización

$\left(P_{k}\right)$

$$
\mid \begin{aligned}
& \text { Min } J_{k}(\omega) \\
& \text { Para todo } \omega \in L^{2}\left(\hat{q}_{T}\right)
\end{aligned}
$$

Por la forma como está definida $J_{k}(\omega)$, resulta semicontinua inferiormente, estrictamente convexa y coerciva en $L^{2}\left(\hat{q}_{T}\right)^{n}$. Entonces existe una solución $\omega_{k}$ de $\left(P_{k}\right)$ para cada $k$.

La derivada de $J_{k}(\omega)$ es definida por

$$
J_{k}^{\prime}(\omega) \cdot \xi=\sum_{i=1}^{n} \int_{\hat{q}_{T}} \omega_{i} \xi_{i} d y d t+k \sum_{i=1}^{n}\left(\left(v_{i \omega}(T)-v_{i d}, v_{i \xi}(\mathrm{T})\right)\right)
$$

para todo $\xi \in L^{2}\left(\hat{q}_{T}\right)^{n}$ donde $\xi=\left(\xi_{1}, \cdots, \xi_{n}\right)$. 
Reemplazando $J_{k}^{\prime}(\omega) \cdot \xi$ en la solución $\omega_{k}$ de $\left(P_{k}\right)$ tenemos

$$
\sum_{i=1}^{n} \int_{\hat{q}_{T}} \omega_{i k} \xi_{i} d x d t+k \sum_{i=1}^{n}\left(\left(v_{i k}(T)-v_{i d}, v_{i \xi}(\mathrm{T})\right)\right)=0
$$

para todo $\xi \in L^{2}\left(\hat{q}_{T}\right)^{n}$ donde $v_{i k}(T)=v_{i} \dot{\omega}_{k}(T)$

Probaremos ahora que $v_{k}(T) \rightarrow v_{d}$ fuerte en $H_{0}^{1}(\Omega)^{n}$.

En efecto, de la minimización tenemos

$$
J_{k}\left(\omega_{k}\right) \leq J_{k}(0)=\frac{k}{2} \sum_{i=1}^{n}\left\|v_{i d}\right\|^{2}
$$

esto es

$$
\frac{1}{2} \sum_{i=1}^{n} \int_{\hat{q}_{T}} \omega_{i k}^{2} d y d t+\frac{k}{2} \sum_{i=1}^{n}\left\|v_{i k}(T)-v_{i d}\right\|^{2} \leq \frac{k}{2} \sum_{i=1}^{n}\left\|v_{i d}\right\|^{2}
$$

De allí

$$
\left(\frac{1}{\sqrt{k}} \omega_{k}\right)_{k \in I N} \text { es acotada en } L^{2}\left(\hat{q}_{T}\right)^{n}
$$

$\mathrm{y}$

$$
\left(v_{k}(T)-v_{d}\right)_{k \in I N} \text { es acotada en } H_{0}^{1}(\Omega)^{n} .
$$

Luego existe una subsucesión que denotamos nuevamente por $\left(v_{k}(T)-v_{d}\right)_{k \in I N}$ tal que $v_{k}(T)-v_{d} \longrightarrow \psi$ débilmente en $H_{0}^{1}(\Omega)^{n}$

donde $\psi=\left(\psi_{1}, \cdots, \psi_{n}\right)$.

De (6) tenemos

$$
\frac{1}{\sqrt{k}} \sum_{i=1}^{n} \int_{\hat{q}_{T}} \frac{1}{\sqrt{k}} \omega_{i k} \xi_{i} d y d t+\sum_{i=1}^{n}\left(\left(v_{i k}(T)-v_{i d}, v_{i \xi}(\mathrm{T})\right)\right)=0
$$

Así, haciendo $k \rightarrow \infty$ y considerando la acotación (8) tenemos

$$
\sum_{i=1}^{n}\left(\left(\psi_{i k}, v_{i \xi}(\mathrm{T})\right)\right)=0 \quad, \quad \forall \xi \in L^{2}\left(\hat{q}_{T}\right)^{n} .
$$

Definimos el: estado adjunto por transposición, sea $p(y, t)=\left(p_{1}(y, t), \cdots, p_{n}(y, t)\right)$, así multiplicando (3) por $p(y, t)$, en seguida integrando por partes tenemos

$$
\int_{Q_{T}} p(y, t)\left[v^{\prime}-\Delta v+a(y, t) v+\vec{b}(y, t) \nabla v\right]=\langle-\Delta \psi, v(T)\rangle
$$


donde $v \in W\left(0, T ; H_{0}^{1}(\Omega)^{n} \cap H^{2}(\Omega)^{n}, L^{2}(\Omega)^{n}\right)$ tal que $v(0)=(0, \cdots, 0) \quad$ y $\quad p$ solución del problema:

$$
\begin{aligned}
& -p^{\prime}-\Delta^{*} p+a^{*}(y, t)-\operatorname{div}\left(\overrightarrow{b^{*}}(y, t) \cdot p\right)=0 \quad \text { en } \quad Q_{T} \\
& p=0 \quad \text { sobre } \quad \sum_{T} \\
& p(T)=-\Delta \psi \quad \text { en } \quad \Omega
\end{aligned}
$$

Observemos que

$$
\begin{aligned}
\int_{Q_{T}} p(y, t) \vec{b}(y, t) \nabla v d y d t=\sum_{\mathrm{i}=1}^{\mathrm{n}} \sum_{\mathrm{j}=1}^{n} \int_{Q_{T}} p_{i} \vec{b}_{i j} \nabla v_{i} d x d t \\
=\sum_{\mathrm{i}=1}^{\mathrm{n}} \sum_{\mathrm{j}=1}^{n} \int_{Q_{T}} v_{i} \operatorname{div}\left(p_{i} \vec{b}_{i j}\right) d x d t \\
=\sum_{\mathrm{i}=1}^{\mathrm{n}} \sum_{\mathrm{j}=1}^{n} \operatorname{div}\left(\vec{b}_{j i} p_{i}\right) \nu_{i} d x d t
\end{aligned}
$$

Consideremos ahora los sistemas

$$
\begin{aligned}
& -p^{\prime}-\Delta^{*} p+a^{*}(y, t)-\operatorname{div}\left(\overrightarrow{b^{*}}(y, t) \cdot p\right)=0 \text { en } Q_{T} \\
& p=0 \quad \text { sobre } \sum_{T} \\
& p(T)=f \quad \text { en } \quad \Omega
\end{aligned}
$$

y

$$
\begin{aligned}
& z^{\prime}-\Delta z+a(y, t)+\vec{b}(y, t) \nabla z=\varphi \text { en } Q_{T} \\
& z=0 \quad \text { sobre } \quad \sum_{T} \\
& z(y, 0)=0 \quad \text { en } \quad \Omega
\end{aligned}
$$

donde $\operatorname{div}\left(\vec{C}_{i j}\right)_{n \times n} \operatorname{denota}\left(\operatorname{div} \vec{C}_{i j}\right)_{n \times n}$.

Si $\varphi \in L^{2}\left(0, T ; L^{2}(\Omega)^{n}\right)$ por el teorema 1 tenemos que $z \in W\left(0, T ; H_{0}^{1}(\Omega) \cap H^{2}(\Omega)^{n}, L^{2}(\Omega)^{n}\right)$ es solución fuerte de (3) (con $\left.v_{0}=0\right)$, desde que $z_{0}=0$ tenemos que $z \in C^{0}\left([0, T] ; H_{0}^{1}(\Omega)^{n}\right) ;$ luego tiene sentido $\langle f, z(T)\rangle$, $\forall f \in H^{-1}(\Omega)^{n}$.

Definición 3: Decimos que p es solución por transposición del problema (13) si $p \in L^{2}\left(0, T ; L^{2}(\Omega)^{n}\right)$ 
y satisface

$$
\int_{Q_{T}} p(y, t) \varphi(y, t) d y d t=\langle f, z(T)>
$$

para todo $\varphi \in L^{2}\left(0, T ; L^{2}(\Omega)^{n}\right)$, donde z es una solución fuerte de (14) correspondiente a $\varphi$.

Teorema 4: Existe una única solución por transposición del sistema (13).

Prueba: Consideremos la forma lineal

$$
L: L^{2}\left(0, T ; L^{2}(\Omega)^{n}\right) \rightarrow I R
$$

definida por $L(\varphi)=<f, z(T)>, \forall \varphi \in L^{2}\left(0, T ; L^{2}(\Omega)^{n}\right), f \in H^{-1}(\Omega)^{n}$ y $z$ solución única fuerte de (14) correspondiente a $\varphi$.

Así tenemos

$$
|<f, z(T)>|=\|f\|_{H^{-1}(\Omega)^{n}}\|z(T)\|_{H_{0}^{1}(\Omega)^{n}}
$$

Por otro lado, ver [4], tenemos

$$
\|z(T)\|_{H_{0}^{1}(\Omega)^{n}} \leq\|z\|_{C^{0}\left([0, T] ; H_{0}^{1}(\Omega)^{n}\right)} \leq C \sum_{i=1}^{n} \int_{0}^{T}\left|\varphi_{i}(t)\right|^{2} d t
$$

luego

$$
|<f, z(T)>|=\|f\|_{H^{-1}(\Omega)^{n}}\|\varphi\|_{L^{2}\left(0, T ; L^{2}(\Omega)^{n}\right)} .
$$

Así $f$ es una forma lineal continua sobre $L^{2}\left(0, T ; L^{2}(\Omega)^{n}\right)$. Por el teorema de representación de Riesz, existe única función

$$
p \in L^{2}\left(0, T ; L^{2}(\Omega)^{n}\right)
$$

tal que

$$
L(\varphi)=\int_{Q_{T}} p(y, t) \varphi(y, t) d y d t
$$

Para todo $\varphi \in L^{2}\left(0, T ; L^{2}(\Omega)^{n}\right)$

Haciendo $f=\Delta \psi \in H^{-1}(\Omega)^{n}$ tenemos que existe una única función $p$ solución por transposición del problema (12), definido por (11), con la siguiente regularidad

$$
p \in L^{2}\left(0, T ; L^{2}(\Omega)^{n}\right) \cap C^{0}\left([0, T] ; H^{-1}(\Omega)\right) \quad(\operatorname{ver}[6])
$$

Sea $v_{\xi}$ solución fuerte de (3) con $\omega=\xi$ es decir el lado derecho de (3) es dado por $\xi \chi_{\hat{q}_{T}} \in L^{2}\left(\Omega_{T}\right)^{n}$. Luego substituyendo en (11) $v$ por $v_{\xi}$ obtenemos

$$
\int_{\hat{q}_{T}} p \xi d y d t=\int_{Q_{T}} p \xi \chi_{\hat{q}_{T}} d y d t=\left(\left(\psi, \nu_{\xi}(\mathrm{T})\right)\right)=0, \forall \xi \in L^{2}\left(\hat{q}_{T}\right)^{n}
$$

dado que $\psi \in H_{0}^{1}(\Omega)^{n}$ y $(10)$. 
Luego $p=(0, \cdots, 0)$ en $\hat{q}_{T}$ es decir $p_{i}=0$ en $\hat{q}_{T}, i=1,2, \cdots, n$.

La primera ecuación del sistema (12) se puede escribir considerando el caso $n=3$ (para visualizar mejor) como :

$$
\begin{aligned}
& -p_{1}^{\prime}-\Delta p_{1}+a_{11} p_{1}-\operatorname{div}\left(\vec{b}_{11} p_{1}\right)=0 \\
& -p_{2}^{\prime}-\Delta p_{1}-\Delta p_{2}+a_{12} p_{1}+a_{22} p_{2}-\operatorname{div}\left(\vec{b}_{12} p_{1}\right)-\operatorname{div}\left(\vec{b}_{12} p_{2}\right)=0 \\
& -p_{3}^{\prime}-\Delta p_{1}-\Delta p_{2}-\Delta p_{3}+a_{13} p_{1}+a_{23} p_{2}+a_{33} p_{3} \\
& -\operatorname{div}\left(\vec{b}_{13} p_{1}\right)-\operatorname{div}\left(\vec{b}_{23} p_{2}\right)-\operatorname{div}\left(\vec{b}_{33} p_{3}\right)=0
\end{aligned}
$$

Así como $p_{1}=0$ en $\hat{q}_{T}$. Se sigue del resultado de unicidad dado en Caroline Fabre [3] que $p_{1}=0$ en $Q_{T}$.

Luego en la segunda línea de (16) tenemos

$$
-p_{2}^{\prime}-\Delta p_{2}+a_{22} p_{2}-\operatorname{div}\left(\vec{b}_{12} p_{2}\right)=0
$$

y como $p_{2}=0$ en $\hat{q}_{T}$ tenemos nuevamente del resultado de unicidad que $p_{2}=0$ en $Q_{T}$.

Finalmente en la última línea de (16) tenemos

$$
-p_{3}^{\prime}-\Delta p_{3}+a_{33} p_{3}-\operatorname{div}\left(\vec{b}_{33} p_{3}\right)=0 \text {, con } p_{3}=0 \text { en } \hat{q}_{T} \text {. }
$$

Nuevamente por el mismo resultado de unicidad tenemos

$$
p_{3}=0 \text { en } Q_{T} \text {. }
$$

Así; $\left(p_{1}, p_{2}, p_{3}\right)=(0,0,0)$ en $Q_{T}$.

En general tenemos

$$
\left(p_{1}, p_{2}, \cdots, p_{n}\right)=(0, \cdots, 0) \text { en } Q_{T} \text {. }
$$

Luego, dado que $p \in C^{0}\left([0, T] ; H^{-1}(\Omega)^{n}\right)$ tenemos que $p(T)=0$. Así $-\Delta \psi=0$ con $\psi \in H_{0}^{1}(\Omega)^{n}$ luego $\psi=0$, por otro lado como de (9) $v_{k}(T)-v_{d} \longrightarrow \quad \psi$ débilmente en $H_{0}^{1}(\Omega)^{n}$ resulta que

$$
v_{k}(T) \longrightarrow v_{d} \text { débilmente en } H_{0}^{1}(\Omega)^{n} .
$$

Haciendo $\xi=\omega_{k}$ en (6) tenemos

$$
\frac{1}{k} \sum_{i=1}^{\mathrm{n}} \int_{\hat{q}_{T}} \omega_{i k}^{2} d y d t=-\sum_{i=1}^{\mathrm{n}}\left(\left(v_{i k}(T)-v_{i d}, v_{i k}(\mathrm{~T})\right)\right) .
$$


De allí tenemos

$$
\frac{1}{k} \sum_{i=1}^{\mathrm{n}} \int_{\hat{q}_{T}} \omega_{i k}^{2} d y d t+\sum_{i=1}^{\mathrm{n}}\left\|v_{i k}(T)-v_{i d}\right\|=-\sum_{i=1}^{\mathrm{n}}\left(\left(v_{i k}(T)-v_{i d}, v_{i d}\right)\right) .
$$

Haciendo $k \rightarrow \infty$ tenemos

$$
v_{i k}(T) \rightarrow v_{i d} \text { fuerte en } H_{0}^{1}(\Omega)
$$

así

$$
v_{k}(T) \rightarrow v_{d} \text { fuerte en } H_{0}^{1}(\Omega)^{n}
$$

\section{Prueba del Teorema 3:}

Sea $u_{d} \in H_{0}^{1}\left(\Omega_{T}\right)^{n}$, definimos $v_{d}(y)=u_{d}(k(T) y)$. Así $v_{d} \in H_{0}^{1}(\Omega)^{n}$, luego por el Lema 3.1 existe una sucesión $\left(v_{k}(T)\right)_{k \in I N} \operatorname{con} v_{k}(T) \in R_{L}(T), k \in I N$ tal que

$$
v_{k}(T) \rightarrow v_{d} \text { fuerte en } H_{0}^{1}(\Omega)^{n}
$$

Sea ahora $u_{k}(x, t)=v_{k}\left(K^{-1}(t) x, t\right)$ luego de la equivalencia de los *problemas (1) y (3) tenemos que $u_{k}(x, t)$ es solución fuerte de (1) para $\hat{\omega}_{k}(x, t)=\omega_{k}\left(K^{-1}(t) x, t\right)$ así

$$
u_{k}(x, T) \in \hat{R}_{L}(T)
$$

Probaremos ahora que $u_{k}(T) \rightarrow u_{d}$ fuerte en $H_{0}^{1}\left(\Omega_{T}\right)^{n}$. En efecto como

$$
\begin{aligned}
\int_{\Omega} \mid \nabla v_{i k}(y, T)- & \left.\nabla v_{i d}(y)\right|^{2} d y \\
& =\int_{\Omega_{T}}\left|\nabla u_{i k}(x, T) k(T)-\nabla u_{i d}(x, T) k(T)\right|^{2}|\operatorname{det} k(T)| d x \\
& =\int_{\Omega_{T}}\left|\nabla u_{i k}(x, T)-\nabla u_{i d}(x, T)\right|^{2} d x \text { puesto que } k(T) \text { es ortogonal. }
\end{aligned}
$$

Luego, haciendo $k \rightarrow \infty$ tenemos

$$
u_{i k} \rightarrow u_{i d} \text { fuerte en } H_{0}^{1}\left(\Omega_{T}\right)
$$

así

$$
u_{k} \rightarrow u_{d} \text { fuerte en } H_{0}^{1}\left(\Omega_{T}\right)^{n}
$$

\section{Agradecimientos:}

Al profesor Manuel Milla Miranda por la sugerencia y acompañamiento del tema y a los profesores J. Limaco y L. A. Medeiros por las constructivas conversaciones. 


\section{REFERENCIAS BIBLIOGRÁFICAS}

1. BREZIS, H. Analyse Fonctionelle (Theory and applications). Masson, Paris, (1983).

2. FABRE, C.; PUEL, J. ; ZUAZUA, E. Approximate controllability of the semilinear heat equation, Proc. Royal Soc. Edinburgh, 125 A, pp 31-61 (1995).

3. FABRE, C. Uniqueness results for Stockes Equations and their consequencias in linear and nonlinear control problems, ESAIM, vol. 1, pp 267-302, (1996).

4. LIONS, J. L. Remark on approximate controllability, Journal d'Analyse Mathématiques, vol. 59, pp 103116, (1992).

5. LIONS, J. L. Sentinelles pour les systemes distribués à données incomplétes, RMA 21, Masson, (1992).

6. LÍMACO, J.; MEDEIROS, L. A. Remarks on approximate controllability in noncylindrical domains, $47^{\circ}$ SBA pp. 1-51 (1998).

7. MILlA MIRANDA, M.; LÍMACO, J. The Navier-Stokes Equations in noncylindrical domain, Comput. Appl. Math. Vol 16, No 3, pp 247-265 (1997). 